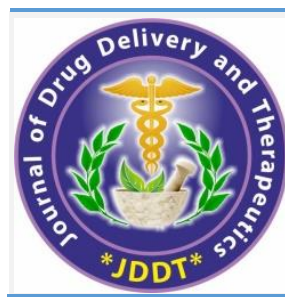

Available online on 15.04.2021 at http://jddtonline.info

\title{
Journal of Drug Delivery and Therapeutics
}

Open Access to Pharmaceutical and Medical Research

(C) 2011-21, publisher and licensee JDDT, This is an Open Access article which permits unrestricted non-commercial use(CC By-NC), provided the original work is properly cited

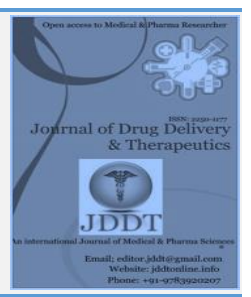

Open Access Full Text Article

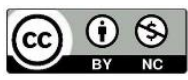

Review Paper

\section{Effect of COVID 19 on thyroid gland function}

\author{
Hussein Adnan Mohammed ${ }^{1 *}$, Saleh Salman Omairi ${ }^{2}$ \\ ${ }^{1}$ Department of Internal Medicine, College of Medicine, Wasit University, Wasit, Iraq \\ ${ }^{2}$ Department of Anatomy and Biology, College of Medicine, Wasit University, Wasit, Iraq
}

\section{Article Info:}

$\begin{array}{ll} & \text { Article History: } \\ & \text { Received 17 Feb 2021 } \\ & \text { Accepted 08 April 2021 } \\ & \text { Available online 15 April } 2021\end{array}$

\section{Cite this article as:}

Mohammed HA, Omairi SS, Effect of COVID 19 on thyroid gland function, Journal of Drug Delivery and Therapeutics. 2021; 11(2-s):195-199

DOI: http://dx.doi.org/10.22270/jddt.v11i2-s.4664

\section{*Address for Correspondence:}

Assist. Prof. Hussein Adnan Mohammed, Department of Internal Medicine, College of Medicin.e, Wasit University, Wasit, Iraq

\section{Abstract}

The pandemic caused by the new Coronavirus (SARS-CoV-2) and the disease induced by it (Coronavirus Disease or COVID-19) has been filling the pages of newspapers, television news and websites for days. It is a new virus, which until a few months ago probably lived only in bats, about which there is little scientific certainty about humans. The few evidences, however, are constantly updated and evolving. It is therefore not uncommon to come across information from unreliable sources and / or without any scientific basis. As far as possible, I will try to shed some light on the possible relationships between the new SARS-CoV-2 coronavirus and the endocrinological system. This article, therefore, is the result of a completely personal research carried out in scientific journals, international scientific research sites and on the opinion of experts in the sector (expert opinions). It does not presume to be exhaustive, as the current certainties are very few and in any case must be interpreted dynamically since the scientific framework is constantly evolving. In essence, the information reported at the time of writing this article is based on what is currently known about the COVID-19 infection, but could be outdated in the coming months, based on the finding of new scientific evidence.

Keywords: Effect, COVID 19, thyroid, gland, function

\section{INTRODUCTION}

The thyroid is central to the body. It's a small gland just under the skin. It lies in front of and next to the larynx and consists of two lobes that resemble a butterfly in shape and are arranged in a crescent shape around the windpipe, just below the larynx. Although it is very small and only weighs around 15-25 grams, it has important tasks in the body and controls our metabolic functions. With the help of iodine, the thyroid gland produces the hormones tetraiodothyronine (T4) and triiodothyronine (T3).(Lee, 2016). These messenger substances cause very specific reactions in the organs: The thyroid regulates oxygen consumption, sugar, fat and protein metabolism and thus the body's energy balance. It is responsible for our heat balance and our body temperature and influences the heart and circulation, the gastrointestinal tract, the muscles and the nervous system. It also regulates the body's mineral and water balance and influences human sexual functions. The entire physical and mental development of children and adolescents also depend on the thyroid gland. (Wei, 2007).

It should be clarified that a virus is not a living organism, but simply genetic material (DNA or RNA), enclosed in a protective envelope (capsid) made up of proteins and sometimes also of lipids. A virus, if absorbed by human cells, is able to modify its genetic code and to take command, starting to replicate and triggering an inflammatory process collaterally. There are different types of viruses, distinguished according to the genetic material (DNA- virus or RNA-virus) or according to the type of protective coating (Rotavirus, Coronavirus, Bacteriophages, Poxvirus etc.). All viruses depend completely on host cells and, as they are non-living beings, they cannot be killed. (Docea, 2020). Fortunately, however, viruses are very fragile, as the only thing that protects them is the thin outer layer of proteins and lipids. This is the reason for the recommendation to wash hands well and frequently, as the use of alcoholic soaps and detergents, dissolving the lipid-protein layer of coating, breaks down the protective capsule and disperses the genetic material contained inside. The spontaneous disintegration time of a virus, on the other hand, is variable and depends on many factors (temperature, humidity, material in which it is found) (Guo, 2019).

\section{LITERATURE REVIEW}

\section{Coronavirus}

The coronavirus (CoV) are RNA viruses (subfamily Orthoronavirinae, Coronaviridae family, suborder Cornidovirineae, Nidovirales order) that can cause various diseases in humans, mainly infections of the upper respiratory tract and gastrointestinal tract. The severity of these conditions is highly variable. In fact, coronaviruses are responsible for both a large part of the common cold syndromes and more serious respiratory syndromes such as SARS (Severe Acute Respiratory Syndrome) and MERS (Middle East Respiratory Syndrome). (Payne, 2017). They owe their name to the appearance of virions under the electron microscope that resembles a royal crown or a solar 
corona. These include: a) Common Human Coronaviruses: 229E (coronavirus alpha), NL63 (coronavirus alpha), OC43 (coronavirus beta), HKU1 (coronavirus beta); b) Other Human Coronaviruses: MERS-CoV (beta coronavirus causing MERS, Middle East respiratory syndrome), SARS-CoV (beta coronavirus causing SARS, Severe acute respiratory syndrome); SARS-CoV-2 novel coronavirus (formerly 2019nCoV). (Britannica, 2021)

\section{- New Coronavirus 2019 (SARS-CoV-2)}

On December 31, 2019, Chinese health authorities reported that an outbreak of atypical pneumonia of unknown etiology had developed in the city of Wuhan (Hubei, central-eastern China), later attributed to a new Coronavirus (SARS-CoV-2). Since then, the epidemic has spread around the world, becoming a pandemic that has involved thousands of people. (Yutaka, 2014). The air route (droplets) is the main mode of transmission of SARS-CoV-2, through contact with symptomatic and asymptomatic infected patients. The incubation period is averaged as between 2 \& 14 days. The onset symptoms are nonspecific, being similar to those of a flu syndrome. In severe cases, it can cause interstitial pneumonia with a typical clinical and radiological picture. (Francesco, 2020). It is not well defined, at the moment, whether there are population groups particularly at risk, but the probability of developing a more serious clinical picture is certainly greater in the elderly and in those with preexisting respiratory, cardiac or metabolic diseases. There appears to be a slight prevalence of cases and mortality in males. Some researchers have hypothesized that this gender prevalence is to be attributed to the different hormonal and immunological structure, which in evolutionary terms favors the survival of the female sex. (Rodriguez, 2020). By comparison with another coronavirus SARS epidemic, the prognosis seems better in youth patients. Children and adolescents very rarely present with severe clinical pictures. (Lu, 2020). From the analysis of Chinese data on COVID-19 mortality in Wuhan, it emerges that the main associated comorbidities are: hypertension (53.8\%), diabetes (42.3\%), heart disease (19.2\%) and cerebral ischemia (15.4\%). These data also seem to be confirmed by the preliminary analysis of the epidemic. The most serious problems from COVID-19 arise in patients who also have other diseases (hypertension, diabetes, cerebral ischemia, Parkinson's disease, renal failure, COPD, autoimmune diseases). Obviously, advanced age and the intake of immune-suppressing drugs (which act on the immune system, inhibiting it) must be added to these risk factors. (Huang, 2019). It should be clarified that patients suffering for example from Rheumatoid Arthritis, Scleroderma, Lupus, etc., do not in themselves have a greater probability of contracting the coronavirus viral infection. However, these patients basically have a functional alteration of the immune system and in addition, frequently; they take therapies (immunosuppressants, steroids, biological drugs) that constantly inhibit the immune response. (Rabi, 2020). For these two reasons, in case of infection, the evolution of the viral disease could be more severe in subjects with autoimmune diseases. It follows that patients with autoimmune disease must strictly follow all the indications given to the general population (hygiene rules, social distancing etc.) but they must not, in any case, independently interrupt or modify the therapy in progress. In the event of a suspected infection or coronavirus, the procedures to be followed are the same as those indicated for the general population. (Singhal, 2020).

- Autoimmune Endocrinological Diseases and COVID19
By knowing close relationship between the immune system and the neuroendocrine system, it is not surprising that numerous autoimmune diseases also directly involve the endocrine glands (thyroid, pancreas, pituitary, adrenal, parathyroid, etc.). Among the most frequent endocrinological autoimmune diseases we mention: Chronic Thyroiditis of Hashimoto, Type 1 Diabetes, Graves ' disease and Addison's Disease. So, another legitimate question is: Do patients with autoimmune endocrinological diseases have an increased risk during COVID-19 infection? As mentioned above, such patients themselves are not more likely to contract the SARS-CoV-2 viral infection. (Kaiser, 2020). In addition, unlike many other autoimmune diseases, endocrinological ones require treatment with substitute drugs and almost never with immune-suppressants that could compromise the immune response and represent a greater risk of vulnerability to infection. (Mattar, 2020).

\section{Thyroid and COVID-19}

In general, there is still no scientific data regarding possible relationships between COVID-19 and thyroid diseases. However, in SARS, another acute respiratory disease caused, however, by another coronavirus, some scientific studies have shown a reduction in the serum levels of thyroid hormones (triiodothyronine and thyroxine), as a possible consequence of damage (inflammatory or hypoxic) that the thyroid suffers during infection. This data was confirmed by a work carried out on (only) five autopsies on patients who died from SARS, which showed an alteration of the normal follicular architecture of the thyroid gland, probably secondary to apoptotic phenomena. This damage to the thyroid parenchyma has been identified both in the follicular cells (thyrocytes) and parafollicular (C cells). This pathological alteration could provide the possible explanation for the low serum levels of triiodothyronine and thyroxine found in patients with SARS. Obviously, whether the SARS (SARS-CoV) data are mutable also for COVID-19 (SARS-CoV-2) is still to be demonstrated. (Dworakowska, 2020).

\section{a) Possible mechanism for COVID-19 entry into thyroid cells identified}

Some researchers started from the well-established information about the role of the Angiotensin Converting Enzyme 2: ACE-2 as the attack molecule of the SARS-CoV-2 virus, which allows the subsequent entry of the latter into the cells of the human organism. ACE-2-expressing cells are also known to be found in many tissues, from those of the lower respiratory tract, to those of the heart and gastrointestinal mucosa. Some authors have confirmed, with laboratory tests, that the presence of ACE- 2 in host cells is essential for the development of SARS-CoV-2 infection and others have found that, by administering antibodiesantiACE-2, it is possible to prevent the entry of the virus even in the cells that present the molecule.( Lu, 2005). The hypothesis that SARS-CoV-2 infection can also be localized in the thyroid is based, first of all, on the identification of viruslike structures in the cells of the thyroid follicles of people with autoimmune thyroiditis. As for the impact of the current SARS-CoV-2 epidemic on the thyroid, clinical cases of subacute thyroiditis have been published in subjects with the infection produced by the virus. (Chakraborty, 2020). Other elements suggesting thyroid involvement in SARSCoV-2 infection are the contiguity of the gland to the higher airways, the main route of entry of the virus into the body, and the observation of thyroid dysfunction in a significant number of people with the infection. Previous research has suggested the presence of ACE- 2 in many cells, including those of the thyroid, but the messenger RNA that serves to 
program the production of ACE- 2 in these cellular elements has not been confirmed (Glinsky,2020). For this reason, Rotondi and colleagues evaluated whether thyroid tissue samples expressed messenger RNA. A laboratory method called Polymerase Chain Reaction (PCR) was used in real time to isolate messenger RNA in thyroid cells obtained from surgically removed glands from people with gland tumors. In particular, the cells of the lobe without neoplasia of glands removed with thyroidectomy interventions were used total. (Li, 2020). The results confirmed the presence of the messenger RNA needed to produce ACE-2 in all thyroid cell samples analyzed. In addition, the transcription level of ACE2 messenger RNA was elevated in both tissue samples and single cells grown in the laboratory. The authors noted that their study showed, for the first time, that thyroid cells express the messenger RNA needed to make ACE-2, which acts as a receptor for SARS-CoV-2. Based on this finding, they concluded that the thyroid could be directly involved in infections caused by this virus and that this would explain the reporting of cases of subacute thyroiditis related to the virus. (Rotondi, 2020).

\section{b) Autoimmune thyroid disease}

The Chronic Hashimoto's thyroiditis is probably the most common endocrine autoimmune disease, as well as the most common cause of hypothyroidism acquired in Western countries. For this pathology, in the absence of development of hypothyroidism, international guidelines do not suggest any pharmacological treatment, and therefore not even with immunosuppressive drugs. Therefore, there are no specific reasons why patients with this disease should have a greater susceptibility to COVID-19 than the general population. (Puig, 2020). There are no specific data on this subject, therefore at the moment, these subjects must be considered and equated to normal subjects of the general population. Also in this case, although it is an autoimmune disease, there is no evidence that patients with Graves' disease constitute a population at risk for COVID-19. Therefore, these patients too should be equated with the general population and should consult their doctor only in case of de-compensation of hyperthyroidism. (Li, 2020).

The thyroid has a very important function in the body: the thyroid hormones regulate the entire basal metabolic rate of the body and do their part to strengthen our immune system. A weak thyroid would also weaken the immune system, which means that viruses and other germs have an easier chance of penetrating the immune system. Even according to the latest findings, people who have thyroid problems do not belong to the risk group when it comes to COVID-19. Hashimoto's thyroiditis or Graves' disease are autoimmune diseases, but in this case the immune system is even slightly increased, which is why these patients are not more susceptible to SARS-CoV-2. However, there are exceptions: People who suffer from thyroid cancer should be careful because, like all cancer patients, they also belong to the risk group. One should also be careful of other diseases that are related to the thyroid gland, such as diabetes mellitus or adrenal insufficiency. (Scappaticcio, 2020).

\section{c) Hypothyroidism}

It is known that thyroid hormones exert an effect on the cells involved in the immune process (monocytes, macrophages, natural killer cells, lymphocytes), and on the various stages of inflammation itself. Therefore, it is questionable whether a patient with a thyroid hormone deficiency (hypothyroidism) can be considered potentially more immune-depressed than a non-hypothyroid subject. (Persani, 2018). However, it should be remembered that the immune system is also regulated by other important extra-thyroidal factors (hormonal and otherwise) which are usually able to guarantee an adequate immune response. Furthermore, it must be understood that a patient with hypothyroidism who correctly takes the replacement therapy with L-Thyroxine, is no longer "technically" hypothyroid and, therefore, is no longer even potentially immune-depressed. (Leow, 2005). In conclusion, the patient with hypothyroidism, adequately treated and compensated, apparently does not present a greater risk of viral infection or complications related to COVID-19, compared to the general population not affected by hypothyroidism. Patients with hypothyroidism in replacement therapy, therefore, are required to follow, like the general population, the general indications dictated by ministerial decrees.

\section{d) Thyroid cancer}

Again there is no published scientific evidence. In general, it can be said that most patients with thyroid cancer are not considered at risk of COVID-19. Only patients who have particularly aggressive or advanced thyroid carcinomas, with widespread metastases, especially in the lungs, or who are taking specific oncological therapies (sorafenib, levantenib, vandetanib) may be at greater risk of developing complications, both for COVID infection. -19, both for possible side effects of the therapies taken. For these patients, therefore, it is advisable to stay at home as much as possible and follow the social distancing procedures even more strictly. (Tsang, 2020).

\section{e) Fine needle aspiration for thyroid nodules during the COVID-19 outbreak}

Tsang et al. have published an article on the management of fine needle aspiration during the COVID-19 epidemic. Their conclusions were that, by applying specific protocols to ensure safety, it was possible to offer this diagnostic procedure to patients who needed it. The COVID-19 epidemic has had serious consequences on the health system. (Tsang, 2020). At the beginning of the spread of the virus in the world, Italy was the second country, after China, by impact of the virus. This has led to the imposition of isolation on the population and to the postponement of all non-urgent medical and surgical procedures. (Vrachimis, 2020). Among these there was also the fine needle aspiration, with the relative cytological analysis, for the thyroid nodules. It is now established that the clinical management of thyroid nodules must be multidisciplinary and, in particular, must be based on a close collaboration between endocrinologists and specialists who analyze the material collected with the fine needle aspirate. This examination should be performed when the ultrasound identified features that indicate a high risk of malignancy for the nodule. On the contrary, when the request for the examination is not appropriate, the fine needle aspiration can be counterproductive, as it leads to unnecessary diagnostic interventions and a lower effectiveness of the diagnostic protocol. In the contingency created by the COVID-19 epidemic, the request for only the examinations that are really necessary has become of fundamental importance, to avoid saturating the services dedicated to them and not to expose staff to unnecessary risks of contact with the virus. (Li, 2020). Palladino and colleagues performed a study aimed at defining how the COVID-19 epidemic has changed clinical practice of the Center in which they operate. The trends in fine needle aspiration exams performed from 1 January 2019 to 13 March 2020, those from 14 March 2020 to 15 May and those from 16 May to 7 July were compared. The total number of tests performed each week dropped dramatically over the three periods from 
a maximum of 62.1 , before the COVID-19 outbreak, to a minimum of 23. The endocrinologists of the Center undertook to define the real priorities to be attributed to the aspirate to be performed, identifying the nodules that, on ultrasound, had shown characteristics at greater risk of malignancy. This commitment in the period of isolation and the limitation to the number of examinations that can be performed has meant that, when normal operations are returned. (Palladino, 2021).

\section{Thyroid patients as a risk group for COVID- 19}

The corona virus SARS-CoV-2, which causes the disease Covid-19, is currently causing a lot of uncertainty. Particular attention is paid to the effects of medication and previous illnesses on the course of the new illness. Thyroid patients also want to know whether they belong to the risk group. Although no data are yet available describing the effects of thyroid disease on the course of Covid-19, in practice there is currently no reason to assume that the risk of a severe course is increased. This applies to the intake of L-thyroxine after radioiodine therapy or surgery, but also to the intake of anti-thyroid drugs (Zhang, 2020). Even patients with an autoimmune thyroid disease, such as Hashimoto's thyroiditis or Graves' disease, do not currently belong to the risk group. The last two cases are autoimmune diseases, but without immunosuppression. Instead, the activity of the immune system tends to be increased. However, caution should be advised if there are additional diseases that are associated with the thyroid gland and pose a risk, such as diabetes mellitus or adrenal insufficiency. Cancers that affect the thyroid gland should also be considered separately due to possible comorbidities, but they do not pose a risk on their own. (Smulever, 2020).

\section{Corona vaccination and thyroid patients}

It is currently frequently asked whether a COVID vaccination for immune diseases of the thyroid gland (Hashimoto's thyroiditis, Graves' disease) or for malignant diseases of the thyroid gland is possible and useful. There is currently no evidence that patients with an autoimmune disease or a (treated) thyroid carcinoma should not be vaccinated. (ETA, 2021). The following information is available on the website of the Austrian Ministry of Social Affairs: The currently available vaccines against COVID-19 have only been partially investigated in people with an autoimmune disease. In any case, data and experience are available on the following diseases: HIV (under therapy and with CD4> 500), stable autoimmune diseases, cancer diseases (without ongoing or recent chemotherapy), diabetes mellitus and cardiovascular and chronic pulmonary diseases before. In all of these cases, no abnormalities regarding efficacy or tolerability were recorded. (Voysey, 2020).

\section{CONCLUSIONS}

1. The thyroid examinations and operations can take place again without restrictions. Nevertheless, it is important to adhere to the safety measures: disinfection of hands before entering the medical facility and wearing mouth and nose protection.

2. The SARS-CoV2 virus, which has been spreading rapidly in China since the beginning of January and now in Europe, is currently leading to major challenges in patient care.

3. In order to protect patients and minimize the risk of infection, it is recommended to avoid social contacts, including visits to ordinations, as far as possible.
4. Regular routine checks are important for thyroid diseases, but in this special situation they can be postponed for a large number of patients without endangering them.

5. In special cases, such as during pregnancy or during the treatment of hyperfunction, it can also make sense in individual cases to send blood findings to the thyroid specialist and then to discuss them by telephone.

6. By applying safety protocols, to prevent the contagion of the people to be examined and of health workers, and by increasing the attention threshold towards performing only the examinations really necessary, it was possible to offer an adequate level of assistance to those who needed fine needle aspiration for thyroid nodules.

7. There is currently no evidence that autoimmune thyroid diseases could be a risk factor for infection with the novel coronavirus COVID-19. Even according to the latest findings, people who have thyroid problems do not belong to the risk group when it comes to COVID-19. Hashimoto's thyroiditis or Graves' disease are autoimmune diseases, but in this case the immune system is even slightly increased, which is why these patients are not more susceptible to SARS-CoV-2. However, there are exceptions: People who suffer from thyroid cancer should be careful because, like all cancer patients, they also belong to the risk group. One should also be careful of other diseases that are related to the thyroid gland, such as diabetes mellitus or adrenal insufficiency.

\section{REFERENCES}

1. Britannica, T. Editors of Encyclopaedia (2021, February 23). Coronavirus. Encyclopedia Britannica. https://www.britannica.com/science/coronavirus-virus-group

2. Chakraborty U, Ghosh S, Chandra A, et al, 2020, Subacute thyroiditis as a presenting manifestation of COVID-19: a report of an exceedingly rare clinical entity; BMJ Case Reports CP; p. 13

3. Docea A.O., Tsatsakis A., Albulescu D., Cristea O., Zlatian O., Vinceti M., Moschos S.A., Tsoukalas D., Goumenou M., Drakoulis N., Dumanov J.M., Tutelyan V.A., Onischenko G.G., Aschner M., Spandidos D.A., Calina D., A new threat from an old enemy: reemergence of coronavirus. Int. J. Mol. Med. 2020.

4. Dworakowska, Dorota \& Grossman, Ashley, 2020, Thyroid disease in the time of COVID-19, Endocrine. 68. 10.

5. ETA-Public Health Board, 2021, Patients with thyroid disease and vaccination against COVID-19, on: https://www.eurothyroid.com/files/download/ETA-PHBVaccination.pdf

6. Francesco Di Gennaro, Damiano Pizzol, Claudia Marotta, Mario Antunes, Vincenzo Racalbuto, Nicola Veronese and Lee Smith, Coronavirus Diseases (COVID-19) Current Status and Future Perspectives: A Narrative Review, Int. J. Environ. Res. Public Health, 2020; 17.

7. Glinsky GV. Tripartite Combination of Candidate Pandemic Mitigation Agents: Vitamin D, Quercetin, and Estradiol Manifest Properties of Medicinal Agents for Targeted Mitigation of the COVID-19 Pandemic Defined by Genomics-Guided Tracing of SARS-CoV-2 Targets in Human Cells, Biomedicines, May 2020; 8(5):129

8. Guo Y.R., Cao Q.D., Hong Z.S., Tan Y.Y., Chen S.D., Jin H.J., Tan K.S., Wang D.Y., Yan Y., The origin, transmission and clinical therapies on coronavirus disease 2019 (COVID-19) outbreakan update on the status, Mil. Med. Res. 2020; 7:11

9. Huang, C.; Wang, Y.; Li, Z.; Ren, L.; Zhao, J.; Hu, Y.; Zhang, L.; Fan, G.; Xu, J.; Gu, X.; et al. Clinical features of patients infected with 2019 novel coronavirus in Wuhan, China. Lancet, 2020; 395:497-506

10. Kaiser U.B., Mirmira R.G., Stewart P.M., Our response to COVID19 as endocrinologists and diabetologists. JCEM 105(5), dgaa148

11. Lee L., Farwell A.P., 2016, Euthyroid sick syndrome, Compr. Physiol., 2020; 15:1071-1080 
12. Leow MK, Kwek DS, Ng AW, Ong KC, Kaw GJ, Lee LS Hypocortisolism in survivors of severe acute respiratory syndrome (SARS). Clin Endocrinol (Oxf). 2005; 63(2):197-202.

13. Li G, Fan Y, Lai Y, et al. Coronavirus infections and immune responses, J Med Virol. 2020; 92:424-32

14. Li M, Marquez RC, Vinales KL, Reaven PD, Behari G, Dildeep A, et al. Considerations for thyroid fine needle aspiration (FNA) biopsies during the COVID-19 pandemic. Clin. Thyroidol. 2020; 32(4):156-158

15. Li M-Y, Li L, Zhang Y, Wang XS, et al. 2020, Expression of the SARS-CoV-2 cell receptor gene ACE2 in a wide variety of human tissues, Infect Dis Poverty;9:45

16. Lu J, Zhou BP, Wen LX, Jiang XL. 2005, [Cloning of ACE-2 gene encoding the functional receptor for the SARS coronavirus and its expression in eukaryotic cells], Zhonghua Shi Yan He Lin Chuang Bing Du Xue Za Zhi, Sep;19(3):260-3, Chinese, PMID: 16261211.

17. Lu, H.; Stratton, C.W.; Tang, Y.W. 2020, Outbreak of pneumonia of unknown etiology in Wuhan, China: The mystery and the miracle. J. Med. Virol., 92, 401-402.

18. Mattar SAM, Koh SJQ, Rama Chandran S, et al. 2020, subacute thyroiditis associated with COVID-19. BMJ Case Rep;13

19. Palladino R, Migliatico I, Sgariglia R, Nacchio M, Iaccarino A, Malapelle U, Vigliar E, Salvatore D, Troncone G, Bellevicine C. Thyroid fine-needle aspiration trends before, during, and after the lockdown: what we have learned so far from the COVID-19 pandemic, Endocrine, 2021 Jan;71(1):20-25.

20. Payne; Susan, 2017, Family Coronaviridae Viruses, 149-158. https://doi.org/10.1016/B978-0-12-803109-4.00017-9

21. Persani L, Brabant G, Dattani M, et al. 2018 European Thyroid Association (ETA) guidelines on the diagnosis and management of central hypothyroidism. Eur Thyroid J., 7(5):225-37.

22. Puig M., Marazuela M., Giustina A., 2020, COVID-19 and endocrine diseases. A statement from the European Society of Endocrinology. Endocrine 68(1), 2-5

23. Rabi, Firas \& Al-Zoubi, Mazhar \& Kasasbeh, Ghena \& Salameh, Dunia \& Al-Nasser, Amjad, 2020, SARS-CoV-2 and Coronavirus Disease 2019: What We Know So Far, Pathogens 9(3)
24. Rodriguez-Morales AJ, Cardona-Ospina JA, Gutiérrez-Ocampo E et al. 2020, Clinical, laboratory and imaging features of COVID19: A systematic review and meta-analysis [published online ahead of print, 2020 Mar 13]. Travel Med Infect Dis.;101623

25. Rotondi, M., Coperchini, F., Ricci, G., Denegri, M., Croce, L., Ngnitejeu, S. T., Villani, L., Magri, F., Latrofa, F., \& Chiovato, L., 2020, Detection of SARS-COV-2 receptor ACE-2 mRNA in thyroid cells: a clue for COVID-19-related subacute thyroiditis, Journal of endocrinological investigation, 1-6

26. Scappaticcio, L., Pitoia, F., Esposito, K. et al. 2020, Impact of COVID-19 on the thyroid gland: an update. Rev Endocr Metab Disord, https://doi.org/10.1007/s11154-020-09615-z

27. Singhal T., 2020, A Review of Coronavirus Disease-2019 (COVID-19), Indian journal of pediatrics, 87(4), 281-286

28. Smulever A, Abelleira E, Bueno F, Pitoia F. 2020, Thyroid cancer in the Era of COVID-19. Endocrine; $1-5$

29. Tsang VH, Gild M, Glover A, Clifton-Bligh R, Robinson BG. 2020, Thyroid cancer in the age of COVID-19. Endocr Relat Cancer

30. Voysey M, Clemens SAC, Madhi SA et al.: 2020, Safety and efficacy of the ChAdOx1 nCoV-19 vaccine (AZD1222) against SARS-CoV-2: an interim analysis of four randomized controlled trials in Brazil, South Africa, and the UK, Lancet

31. Vrachimis A, Iakovou I, Giannoula E, Giovanella L. ENDOCRINOLOGY IN THE TIME OF COVID-19: management of thyroid nodules and cancer. Eur. J. Endocrinol. 2020;183(1)

32. Wei L., Sun S., Xu C.H., Zhang J., Xu Y., Zhu H., Peh S.C., Korteweg C., McNutt M.A., Gu J., 2007, Pathology of the thyroid in severe acute respiratory syndrome. Hum. Pathol. 38, 95-102

33. Yutaka Terada, Nobutaka Matsui, Keita Noguchi, Ryusei Kuwata, Hiroshi Shimoda, Takehisa Soma, Masami Mochizuki, Ken Maeda, Emergence of pathogenic coronaviruses in cats by homologous recombination between Feline and Canine coronaviruses, PLoS One, 2014:9, p. 9

34. Zhang D, Fu Y, Zhou L, et al. 2020, Thyroid surgery during coronavirus-19 pandemic phases I, II and III: Lessons learned in China, South Korea, Iran and Italy. J Endocrinol Invest. 